EPJ Web of Conferences 66, 02036 (2014)

DOI: 10.1051/epjconf/2014660 2036

(C) Owned by the authors, published by EDP Sciences, 2014

\title{
Spectroscopic Tools Applied to Element Z = 115 Decay Chains
}

U. Forsberg1,a, D. Rudolph ${ }^{1}$, P. Golubev ${ }^{1}$, L.G. Sarmiento1, A. Yakushev², L.-L. Andersson ${ }^{3}$, A. Di Nitto ${ }^{4}$, Ch.E. Düllmann" ${ }^{2,3,4}$, J.M. Gates ${ }^{5}$, K.E. Gregorich ${ }^{5}$, C.J. Gross ${ }^{6}$, F.P. Heßberger ${ }^{2,3}$, R.-D. Herzberg ${ }^{7}$, J. Khuyagbaatar ${ }^{2}$, J.V. Kratz ${ }^{4}, K^{2}$ Rykaczewski ${ }^{6}$, M. Schädel ${ }^{2,8}$, S. Åberg ${ }^{1}$, D. Ackermann ${ }^{2}$, M. Block ${ }^{2}$, H. Brand ${ }^{2}$, B.G. Carlsson ${ }^{1}$, D. Cox ${ }^{7}$, X. Derkx ${ }^{3,4}$, K. Eberhardt ${ }^{3,4}$, J. Even ${ }^{3}$, C. Fahlander ${ }^{1}$, J. Gerl², E. Jäger ${ }^{2}$, B. Kindler ${ }^{2}$, J. Krier², I. Kojouharov², N. Kurz², B. Lommel ${ }^{2}$, A. Mistry ${ }^{7}$, C. Mokry ${ }^{3,4}$, H. Nitsche ${ }^{5}$, J.P. Omtvedt ${ }^{9}$, P. Papadakis ${ }^{7}$, I. Ragnarsson ${ }^{1}$, J. Runke ${ }^{2}$, H. Schaffner ${ }^{2}$, B. Schausten ${ }^{2}$, P. Thörle-Pospiech ${ }^{4}$, T. Torres ${ }^{2}$, T. Traut ${ }^{4}$, N. Trautmann $^{4}$, A. Türler ${ }^{10}$, A. Ward ${ }^{7}$, D.E. Ward ${ }^{1}$, and N. Wiehl ${ }^{3,4}$

${ }^{1}$ Department of Physics, Lund University, S-22100 Lund, Sweden

${ }^{2}$ GSI Helmholzzentrum für Schwerionenforschung GmbH, D-64291 Darmstadt, Germany

${ }^{3}$ Helmholtz Institute Mainz, D-55099 Mainz, Germany

${ }^{4}$ Johannes Gutenberg-Universität Mainz, D-55099, Germany

${ }^{5}$ Nuclear Science Division, Lawrence Berkeley National Laboratory, Berkeley, CA 94720, USA

${ }^{6}$ Physics Division, Oak Ridge National Laboratory, Oak Ridge, TN 37831, USA

${ }^{7}$ University of Liverpool, Liverpool L69 7ZE, United Kingdom

${ }^{8}$ Advanced Science Research Center, Japan Atomic Energy Agency, Tokai, Japan

${ }^{9}$ University of Olso, NO-0315 Oslo, Norway

${ }^{10}$ Paul Scherrer Institute and University of Bern, $\mathrm{CH}-5232$ Villigen, Switzerland

\begin{abstract}
Nuclides that are considered to be isotopes of element $Z=115$ were produced in the reaction ${ }^{48} \mathrm{Ca}+{ }^{243} \mathrm{Am}$ at the GSI Helmholtzzentrum für Schwerionenforschung Darmstadt. The detector setup TASISpec was used. It was mounted behind the gas-filled separator TASCA. Thirty correlated $\alpha$-decay chains were found, and the energies of the particles were determined with high precision. Two important spectroscopic aspects of the offline data analysis are discussed in detail: the handling of digitized preamplified signals from the silicon strip detectors, and the energy reconstruction of particles escaping to upstream detectors relying on pixel-by-pixel dead-layer thicknesses.
\end{abstract}

\section{Introduction}

In November 2012, the TASISpec setup [1] was employed in an experiment aiming at detailed spectroscopic studies of superheavy elements occurring in decay chains originating from the fusionevaporation reaction ${ }^{48} \mathrm{Ca}+{ }^{243} \mathrm{Am}$. TASISpec comprises five double-sided silicon strip detectors (DSSSDs) in the shape of a box with one side left open for incoming ions. The nuclei of interest are separated from the primary beam and guided to the setup using the TASCA separator [2-4], and implanted into the central DSSSD ('implantation detector'). Their subsequent charged-particle decays are detected in it and the surrounding silicon detectors ('upstream detectors'). Coincident photons are measured in five surrounding, large composite germanium detectors [1].

During 18 days of beam time, 30 correlated alpha decay chains were detected [5]. Their $\alpha$-decay characteristics are by and large consistent with previously published data on element 115 chains [6].

\footnotetext{
a e-mail: Ulrika.Forsberg@ nuclear.lu.se
}

This is an Open Access article distributed under the terms of the Creative Commons Attribution License 2.0, which permits unrestricted use, distribution, and reproduction in any medium, provided the original work is properly cited. 
Observed $\alpha$-photon coincidences allow for construction of level schemes and provide candidates for characteristic X-rays along the ${ }^{288} 115$ decay chain $[5,7]$. To achieve the necessary high spectroscopic energy precision, several measures were taken. Using digital electronics, pulse shapes were stored and processed offline for optimal energy determination. Reconstruction of energy loss of escaping $\alpha$ particles passing through detector dead layers was done on a pixel-by-pixel basis. The detectors were calibrated using various standard radioactive sources. In-beam data was used to account for possible small drifts of the amplification stages of electronic channels during the experiment. Here, the methods used in processing the pulse shapes, the methods used for pixel-by-pixel determination of the dead layer, and the routine for reconstructing the energy of the decay when it is split between the implantation detector and an upstream detector, are described.

\section{Pulse Shape Analysis}

Signals from all 192 silicon detector strips were processed using charge-sensitive preamplifiers developed at the University of Cologne [8]. Signals from the n-doped sides were processed in standard shaping amplifiers and ADCs, while those from the p-doped sides were digitized using 60-MHz 12 bit FEBEX sampling ADCs [9]. Pulse shapes of $\sim 70 \mu$ s length were recorded after each accepted trigger. The trigger condition was defined by a coincident signal in a p-side and an n-side strip in the implantation detector. The traces were then analyzed offline using methods similar to those described in e.g. [10]. A typical pulse shape is shown in Fig. 1. The decay constant for the preamplifiers is nominally $13 \mu \mathrm{s}$, but varies for the individual devices within $\pm 1 \mu \mathrm{s}$. A moving window deconvolution (MWD) algorithm was used to extract the energy of each pulse. As the decay constant of the preamplifier is used as a parameter in this algorithm, it was individually determined for each preamplifier channel by least-square fitting an exponential function to the relevant portion of the traces. To be less sensitive to small temporal changes in the decay constant and to even out noise, several pulses recorded during a long $\alpha$ source calibration were added prior to this fitting. Some constraints were imposed on the pulses to be summed, such as requiring that the pulse arrived at a time suggesting that the event was self-triggered, heights corresponding to full-energy $\alpha$-particle detection, and that it had no pile-up of overlayed pulses. These constraints were realized by applying a short trapezoidal filter on the data. The resulting decay constants were used in the MWD algorithm. The algorithm was applied to each stored pulse shape, and the resulting energies were used for further analysis.

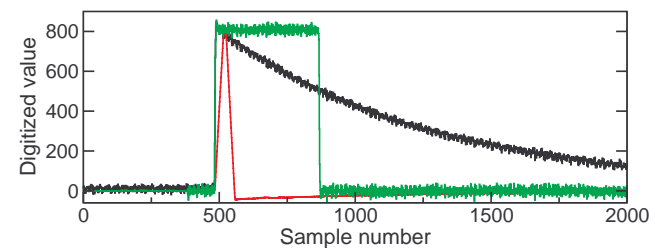

Figure 1. A typical pulse shape with a single $\alpha$ shown in black together with the results after application of the short trapezoidal filter (red) and the MWD filter (green).

\section{Energy reconstruction of particles escaping to upstream detectors}

In about $50 \%$ of the decays of the implanted nuclei, the emitted particles are directed into the implantation detector. This means that the full energy released in the decay, i.e. both the energy of the emitted particle and the recoil energy of the mother nucleus, is detected in the implantation DSSSD. In the other cases, the particle escapes from the implantation detector, leaving only the recoil energy and some fraction of the particle energy in this detector. With a probability of about $60 \%$, the escaping particle is registred in one of the upstream detector. In Fig. 2(a), this situation is shown schematically. In principle, it is possible to sum the two energies to get the full energy released in the decay. A problem with this method arises due to the dead layers on the DSSSDs, because the energy loss in these inactive volumes can be several hundreds of $\mathrm{keV}$ for the $\alpha$ particles of interest. 
To compensate for this energy loss, as well the dead layer thickness as knowledge about the particle's trajectory are needed. Due to the pixellation of the TASISpec DSSSDs, it is possible to calculate the angle at which the two dead layers (one on the implantation detector and one on the upstream detector) are traversed. Given this information, the energy released in a decay can be reconstructed.

Using SRIM [11], the energy loss $(\mathrm{d} E / \mathrm{d} x)$ as a function of $\alpha$-particle energy can be calculated. A routine was developed that starts from the energy measured in the upstream detector and iterates 'backwards' through the total dead layer in steps of a few hundredths of a micrometer. In each step, the relevant energy loss is evaluated and added to the total energy of the particle. This procedure is repeated until the beginning of the dead layer is reached. Then, the energy measured in the implantation detector is added to yield the total energy released in the decay. Unfortunately, there are some uncertainties in this method. Since the pixels are not infinitely small, the angle at which the dead layers are traversed cannot be exactly determined. In addition, the thicknesses of the dead layers across the DSSSDs are not uniform and they have not previously been accurately measured. Thus, they have to be individually determined for each pixel.

To determine the pixel-by-pixel dead layer thickness, long calibration runs in conjunction with GEANT4 simulations of the setup [12] were used. The main idea is to calibrate the detectors with electron sources (as the results are essentially independent of the dead layer) and implanted background activity, extract the positions of $\alpha$ peaks from standard radioactive sources for each pixel in the array, and then compare the experimental peak positions with simulated peak positions. For these purposes, radioactive sources containing ${ }^{207} \mathrm{Bi}$ (electrons, $975.7 \mathrm{keV}$ and $\left.481.7 \mathrm{keV}\right)$ and ${ }^{148} \mathrm{Gd}(\alpha$, $3.18 \mathrm{MeV}$ ) were used. The dead layer used in the simulations is varied until the peak positions agree. For the implantation detector, this could be done in a few iterations by visual inspection. The dead
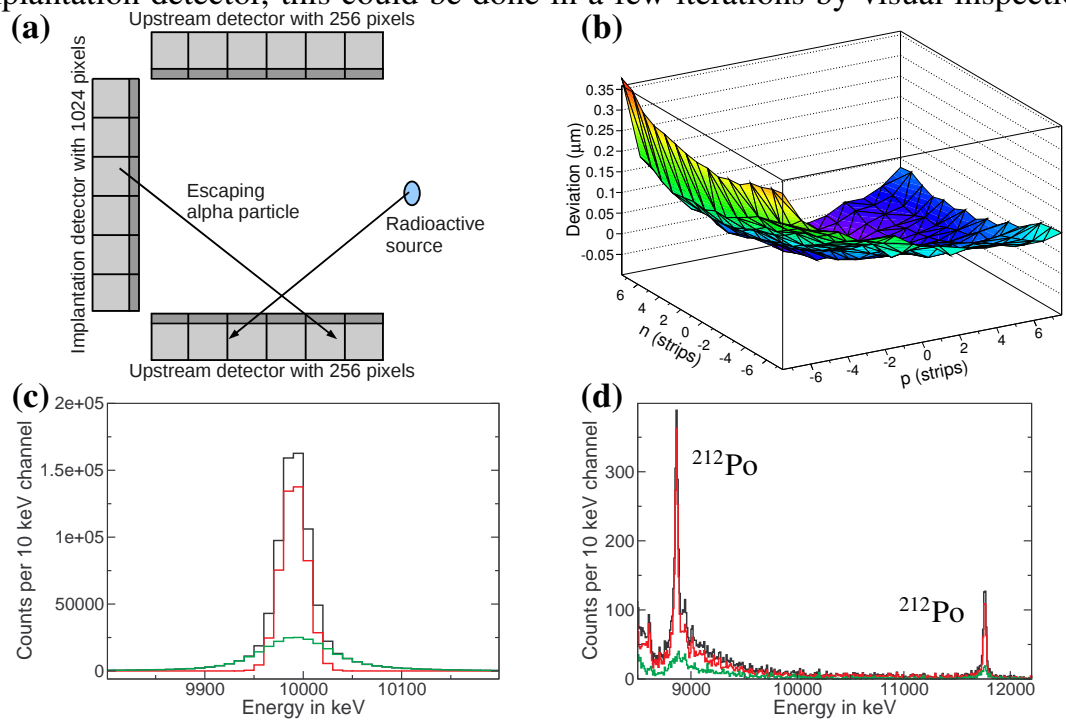

Figure 2. A sketch of a part of the experimental setup is shown in panel (a); the leftmost detector is the implantation detector, and the others represent two of the upstream detectors. An $\alpha$ particle escaping from the implantation detector passes through two dead layers, while a particle from the radioactive source passes through only one (assuming the source has no dead layer). Both the dead layer of the specific pixels involved and the angle between them are important. Panel (b) shows the difference between the simulated constant dead layer and the deduced dead layer, before fitting a function over the pixels to smoothen the surface to a physically acceptable shape. The energy spectra show the results of the reconstruction routine applied to simulated (c) and experimental in-beam (d) data. Spectra shown in black are from all the events, those in red are from the implantation detector only, and the green spectra are from reconstructed events only. The summed spectrum from all events in (d) has a FWHM of $33(2) \mathrm{keV}$ at $11.8 \mathrm{MeV}$. 
layer was found to agree well with a layer of approximately $2 \mu \mathrm{m} \mathrm{SiO} \mathrm{S}_{2}$, with a smooth surface described by a quadratic function $f(x, y)$ with different coefficients for the $x^{2}$ and $y^{2}$ terms allowed in positive and negative directions starting from the center of the detector.

For the upstream detectors, an automatic routine was implemented. The peak positions were simulated for a constant dead layer of $2.3 \mu \mathrm{m}$ and compared to measured peak positions for each pixel. If the energies did not match, the appropriate amount of dead layer to add or to remove was estimated. This estimate was based on the difference in simulated and measured energy and the mean angle of incidence. It took into account that the relevant energy loss in the small portion of added or removed dead layer is energy dependent. Figure 2(b) shows the difference between simulated and deduced dead layer thickness for one of the upstream detectors. In a final step, a function of the same type that was found suitable for the implantation detector was fitted to the data, and pixel-by-pixel dead layers were extracted from this smooth surface.

Using the deduced dead layer thickness, the reconstruction routine was applied to events in which the energy was divided between the implantation detector and an upstream detector. The result is exemplified in Fig. 2 for both simulated (c) and experimental (d) in-beam data, and compared with the energy spectra taken solely with the implantation detector, and with the sum of all events. The resolution is naturally somewhat worse for the reconstructed events compared to those in which the full energy is released in the implantation detector, but the method indeed reconstructs the peaks at the right positions.

\section{Summary and conclusions}

To optimize DSSSD energy resolution from pulse shapes stored during a superheavy element spectroscopy experiment, a specific MWD algorithm fine-tuned with individually optimized decay constants was worked out. Furthermore, pixel-by-pixel dead-layer thicknesses of TASISpec DSSSDs were determined to optimize the reconstruction of the energies of $\alpha$ particles escaping the implantation detector and entering the upstream detectors. Together with other methods described in [7], high spectroscopic precision in our measurements is achieved for the $\alpha$-particle range of interest (8$12 \mathrm{MeV}$ ): uncertainty of a single energy measurement is $10 \mathrm{keV}$ in case the energy measurement was confined in the implantation detector, and 40-100 keV (depending on actual dead layer thickness) when the $\alpha$ particle left part of its energy in one of the upstream detectors.

\section{Acknowledgments}

The authors would like to thank the ion-source and the accelerator staff at GSI. This work is supported by the European Community FP7 - Capacities ENSAR No. 262010, the Royal Physiographic Society in Lund, the Swedish Research Council, the German BMBF, the Office of Nuclear Physics, U.S. Department of Energy, and the UK Science and Technology Facilities Council.

\section{References}

[1] L.-L. Andersson et al., Nucl. Instr. Meth. A622, 164 (2010).

[2] A. Semchenkov et al., Nucl. Instr. Meth. B 266, 4153 (2008).

[3] M. Schädel, Eur. Phys. J. D 45, 67 (2007).

[4] J. M. Gates et al. Phys. Rev. C. 83, 054618 (2011).

[5] D. Rudolph et al., Phys. Rev. Lett. 111, 112502 (2013).

[6] Yu. Ts. Oganessian et al., Phys. Rev. C 87, 014302 (2013).

[7] U. Forsberg et al., to be published.

[8] P. Golubev et al., Nucl. Instr. Meth. A723, 55 (2013).

[9] J. Hoffmann et al., GSI Scientific Report 2011, GSI Report 2012-1 (2012).

[10] L. Arnold et al., IEEE Trans. Nucl. Sci. 53, 723 (2006).

[11] www.srim.org

[12] L.G. Sarmiento, L.-L. Andersson, D. Rudolph, Nucl. Instrum. Meth. A667, 26 (2012). 